\author{
ОЦЕНКА СЕЙСМОНАПРЯЖЕННОГО СОСТОЯНИЯ \\ ОБДЕЛОК ТОННЕЛЕЙ МЕЛКОГО ЗАЛОЖЕНИЯ \\ П. В. Деев, М. А. Петрухин \\ Тульский государственный университет, \\ E-mail: dodysya@yandex.ru,np-m Ленина 92, г. Тула 300012, Россия,
}

\begin{abstract}
Предложен аналитический метод расчета многослойных обделок тоннелей мелкого заложения на сейсмические воздействия землетрясений, позволяющий учитывать влияние сейсмических волн, отраженных от земной поверхности, и волн Рэлея. В результате расчета для каждого радиального сечения обделки тоннеля определяется наиболее опасное напряженное состояние, которое может иметь место при землетрясении заданной интенсивности. В качестве примера рассмотрена обделка тоннеля, расположенного в сейсмически активном районе. Построены огибающие эпюр напряжений на внутреннем контуре поперечного сечения обделки по максимальным значениям сжимающих и растягивающих напряжений, а также эпюры продольных сил и изгибающих моментов. Получены и проанализированы зависимости максимальных напряжений, возникающих в обделке тоннеля при землетрясении, от глубины заложения.
\end{abstract}

Тоннель, обделка, напряженное состояние, землетрясение, расчет

\title{
STRESS STATE OF LINING IN SHALLOW TUNNELS SUBJECTED TO SEISMIC EFFECT
}

\author{
P. V. Deev and M. A. Petrukhin \\ Tula State University, E-mail: dodysya@yandex.ru, \\ ul. Lenina 92, Tula 300012, Russia
}

\begin{abstract}
A new analytical method is proposed for seismic analysis of multilayer linings in shallow tunnels. The method allows taking into account the influence of seismic waves reflected from the Earth's surface and Rayleigh waves. The most hazardous stress state that may take place in radial sections of the lining during the earthquake of given intensity is determined. As an example, the seismic analysis is performed for the lining of a tunnel located in a seismically active area. The envelope diagrams of maximal values of tensile and compressive stresses along internal outline of the lining cross section and corresponding diagrams of longitudinal forces and bending moments in the lining are constructed. The dependences of the maximum stresses arising in the lining during the earthquake on the tunnel depth are obtained and analyzed.
\end{abstract}

Tunnel, lining, stress state, earthquake, design

Анализ данных о воздействии разрушительных землетрясений на подземные конструкции в работах [1-3] позволил выявить факторы, существенно влияющие на вероятность повреждения обделки тоннеля при землетрясении. В частности, установлено, что тоннели мелкого заложения более уязвимы для сейсмических воздействий землетрясений, чем подземные сооружения на значительной глубине.

Нормативные документы, регламентирующие расчет тоннелей, сооружаемых в сейсмически активных районах $[4,5]$, рекомендуют использовать для оценки напряженного состояния подземных конструкций аналитические решения плоских квазистатических задач теории упругости. 
Подземное сооружение моделируется кольцом, в общем случае, произвольной формы, подкрепляющем отверстие в линейно-деформируемой среде, моделирующей массив грунта. Напряжения, прикладываемые к среде на бесконечности, моделируют сейсмическое воздействие землетрясения. Величина напряжений находится из условия равенства максимального ускорения частиц грунта нормативному значению ускорения, соответствующему баллу землетрясения. В результате для каждого рассматриваемого нормального сечения подземной конструкции рассчитывается наиболее неблагоприятное напряженное состояние, которое может возникнуть при землетрясении [6].

В работах $[7,8]$ предложено использовать описанный подход для расчета обделок тоннелей мелкого заложения. Массив пород представлялся линейно-деформируемой полуплоскостью, нагруженной вдоль границы и на бесконечности напряжениями, моделирующими сейсмическое воздействие, определяемыми таким же образом, как и при расчете тоннелей глубокого заложения. В работе [9] при расчете подземных сооружений, расположенных на небольшой глубине, учитывается влияние сейсмических волн, отраженных от земной поверхности. Обделка тоннеля моделируется многослойным кольцом, подкрепляющим отверстие в линейно-деформируемой полуплоскости, нагруженной вдоль границы и на бесконечности напряжениями, моделирующими сейсмическое воздействие, которые вычисляются благодаря решению задачи о распространении плоских волн в упругой полуплоскости. Подход в [9] позволяет находить максимальные напряжения, появляющиеся в обделке тоннеля при распространении в массиве продольных и поперечных волн, а также поверхностных волн Рэлея.

Следует отметить, что указанные методы применимы только к расчету подземных конструкций, сооружаемых закрытым способом, без вскрытия земной поверхности. Для тоннелей, пройденных открытым способом, необходимы методы, учитывающие специфику взаимодействия таких конструкций с массивом грунта при землетрясении.

Используемый в настоящее время подход к расчету подземных сооружений на сейсмические воздействия землетрясений [6] предусматривает случай, когда растягивающие напряжения, возникающие в массиве грунта, не передаются на подземную конструкцию и расчет на действие продольной волны в фазе растяжения не проводится. При отражении волн от границы полуплоскости использовать такой прием не представляется возможным, поэтому в работе [9] для конструкций, в которых допускается образование трещин при землетрясении, при определении растягивающих напряжений предлагается рассматривать только случаи, в которых максимальное по абсолютной величине главное напряжение в точке полуплоскости, соответствующей положению центра сечения тоннеля, является сжимающим.

Приведем пример расчета обделки, поперечное сечение которой показано на рис. 1, на сейсмические воздействия землетрясений. Исходные данные для расчета принимались следующими: глубина заложения тоннеля $H=10$ м, объемный вес грунта $\gamma=0.02 \mathrm{MH} / \mathrm{m}^{3}$, деформационные характеристики грунта $E_{0}=500 \mathrm{MПа,} v_{0}=0.3$, характеристики бетона $E_{1}=30000 \mathrm{MПа,} v_{1}=0.2$. Ребра тюбингов моделировались слоем с приведенным модулем деформации $E_{2}=9000$ МПа (коэффициент армирования $\mu=0.3$ ) и коэффициентом Пуассона $v_{2}=0.2$. В соответствии с подходом, представленным в [6], критерием того, что напряженное состояние рассматриваемого радиального сечения обделки тоннеля является наиболее опасным, является величина напряжений в соответствующей точке внутреннего контура поперечного сечения конструкции. Совокупность максимальных сжимающих или растягивающих напряжений, которые могут возникнуть на внутреннем контуре поперечного сечения тоннеля при сейсмическом воздействии, называется огибающей эпюр напряжений по максимальным сжимающим или растягивающим напряжениям. 

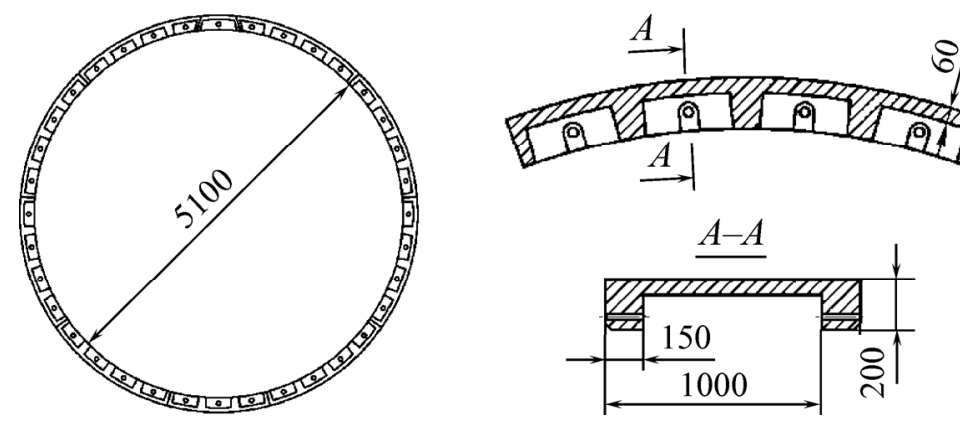

Рис. 1. Поперечное сечение обделки рассматриваемого тоннеля

На рис. 2 изображены огибающие эпюр по максимальным значениям сжимающих (сплошные линии) и растягивающих (пунктир) напряжений в ребрах тюбингов при действии продольной волны (рис. 2a), поперечной волны (рис. 2б) и волны Рэлея (рис. 2в). В силу симметрии полученного распределения напряжений даны только половины эпюр. Напряжения в обделке тоннеля приведены в долях максимальных напряжений, возникающих в бесконечной среде при распространении соответствующих волн (напряжений во фронте волны).

Огибающая, построенная по максимальным значениям напряжений, выбранным из трех указанных эпюр, показана на рис. 22. При ее построении напряжения во фронте каждой из рассматриваемых волн определялись исходя из нормативного значения ускорения, соответствующего интенсивности расчетного землетрясения. Получены следующие расчетные значения на-

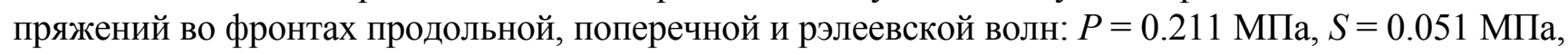
$P_{R}=0.187$ МПа. В отличие от подхода, предложенного в [6], напряжения, найденные при действии волн разных типов, не складывались, так как это привело бы к превышению нормативных значений ускорений.

На рис. $2 \partial, e$ представлены расчетные эпюры усилий в обделке тоннеля. Для их построения напряжения на остальных контурах поперечных сечений слоев обделки определялись при действии тех типов волн, том направлении волн и в тот момент времени, при которых получены максимальные напряжения на внутреннем контуре. По вычисленным значениям напряжений найдены продольные силы (рис. 2d) и изгибающие моменты (рис. 2e) в обделке тоннеля. Очевидно, что для оценки прочности подземной конструкции полученные значения усилий необходимо суммировать с результатами расчета на основные виды нагрузок.

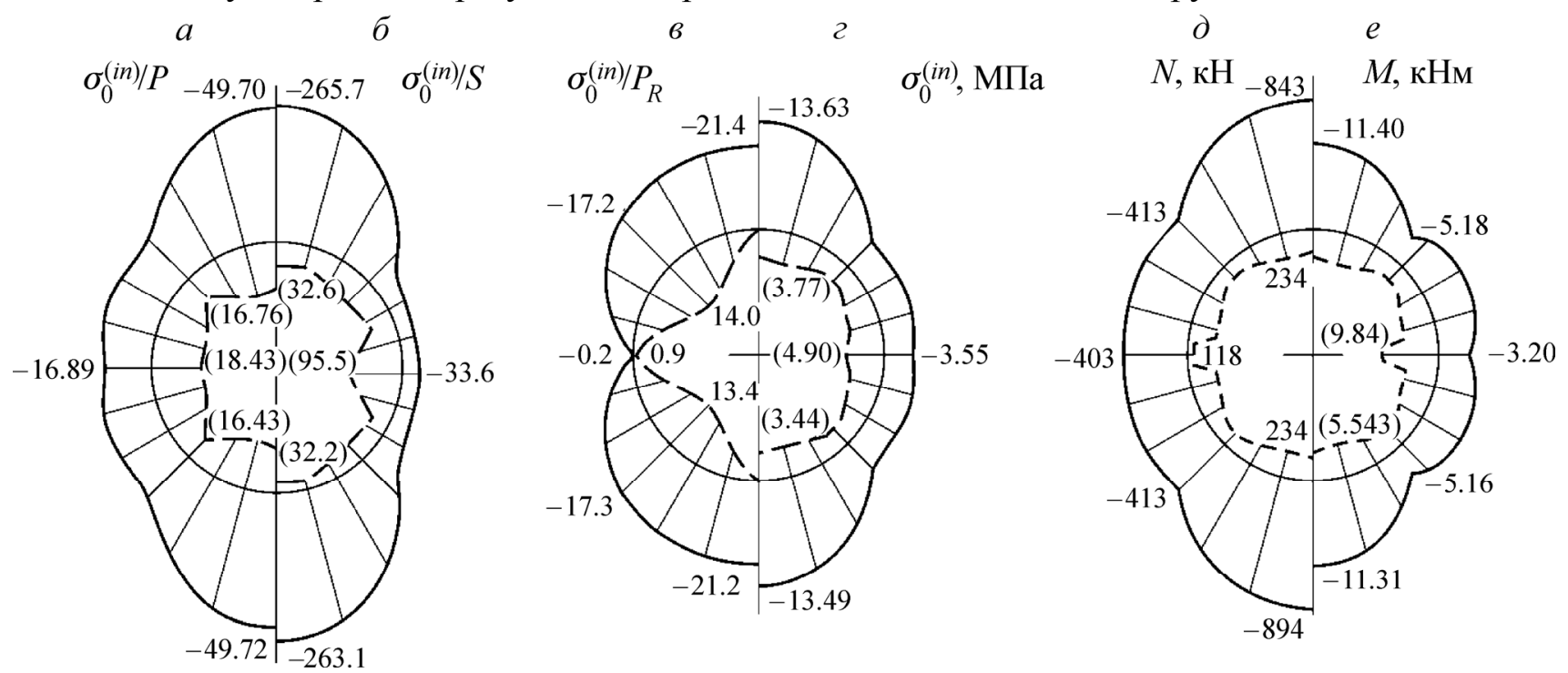

Рис. 2. Результаты расчета: максимальные напряжения от действия продольной (a), поперечной (б) и рэлеевской (в) волн; 2 - суммарные напряжения; $\partial-$ продольные силы; $e-$ изгибающие моменты 
Видно, что при действии продольной и поперечной волн максимальные сжимающие напряжения возникают в своде и лотке, а максимальные растягивающие - в стенках обделки. При действии волн Рэлея максимальные сжимающие напряжения появляются в своде и лотке обделки тоннеля, максимальные растягивающие - на участках внутреннего контура, прилегающих к диаметру, наклоненному под углом $45^{\circ}$ к горизонтали. Наиболее опасно для обделки тоннеля действие поперечных волн. Максимальные усилия обнаружены в своде и лотке обделки тоннеля. Распределение напряжений и усилий в обделке рассматриваемого тоннеля свидетельствует о том, что преимущественным воздействием на подземную конструкцию, расположенную на небольшой глубине, является горизонтальное сжатие, возникающее в массиве грунта при землетрясении.

На основе данных многовариантных расчетов построены зависимости максимальных сжимающих и растягивающих напряжений в обделке тоннеля от глубины заложения. На рис. 3 показаны зависимости максимальных растягивающих $\sigma_{\theta}^{(\max , t)}$ и сжимающих $\sigma_{\theta}^{(\max , c)}$ окружных напряжений, полученные при действии продольных (рис. $3 a$ ), поперечных (рис. 3б) и рэлеевской (рис. 3в) волн. Цифрами обозначены кривые, соответствующие значениям коэффициента Пуассона грунта: $1-0.2 ; 2-0.3 ; 3-0.4$.
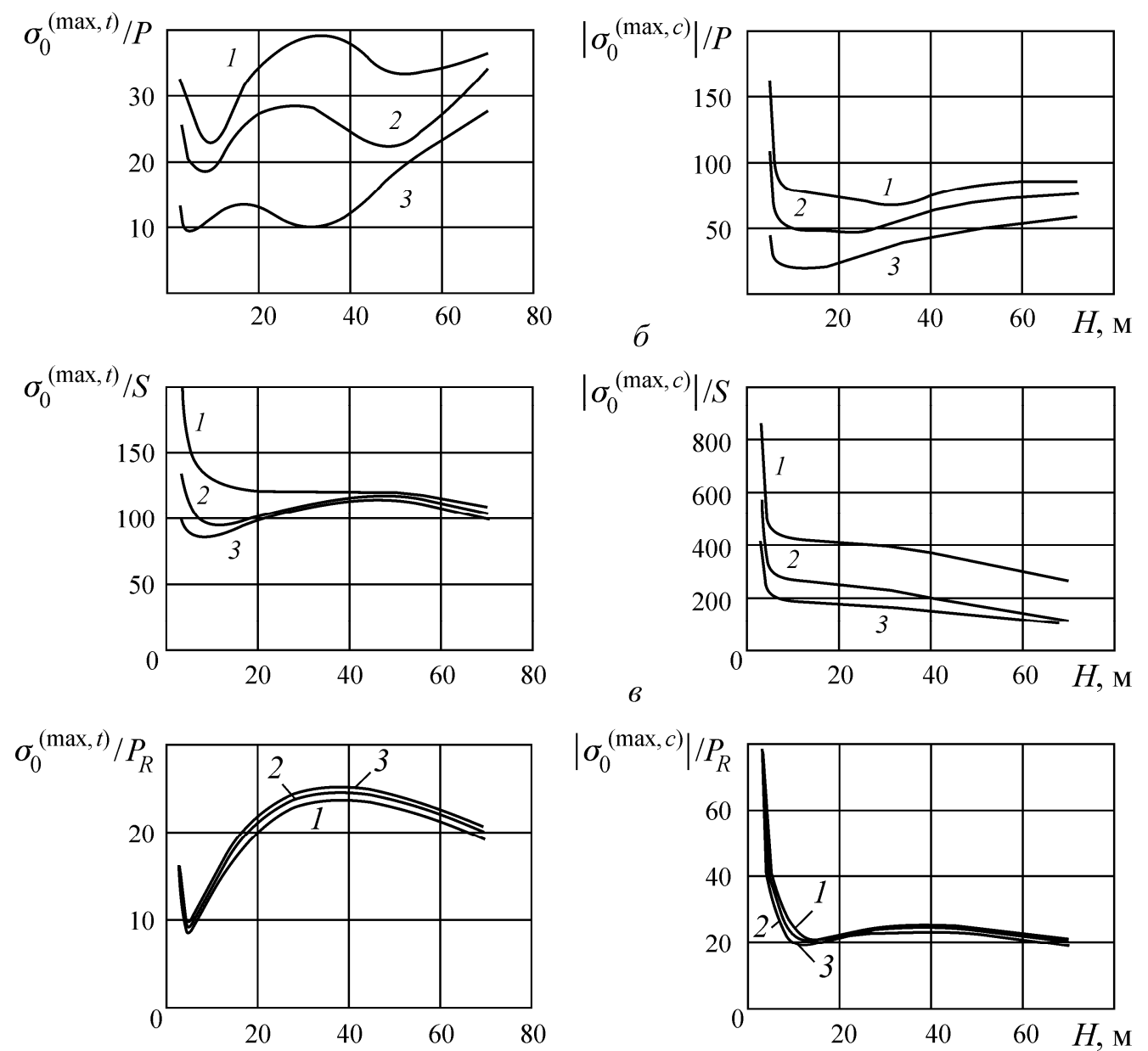

Рис. 3. Зависимости максимальных сжимающих и максимальных растягивающих напряжений в обделке тоннеля от глубины заложения при действии: $a-$ продольных волн; $\sigma-$ поперечных волн; в - волн Рэлея

Из анализа представленных зависимостей можно сделать следующие выводы:

- при глубине заложения тоннеля менее двух диаметров выработки влияние земной поверхности приводит к значительному возрастанию расчетных напряжений; 
— наибольшая концентрация напряжений наблюдается в случае распространения поперечных волн;

- зависимости растягивающих напряжений от глубины заложения тоннеля имеют явно выраженные локальные максимумы и минимумы;

- с увеличением глубины заложения напряжения, вызванные действием продольных волн, в целом, возрастают, поперечных волн и волн Рэлея - убывают.

Для формирования общей картины изменения напряженного состояния подземной конструкции с увеличением глубины заложения тоннеля для каждого значения $H$ определены максимальные напряжения, которые могут возникнуть в обделке при девятибалльном землетрясении. Из напряжений, полученных при действии продольной, поперечной и рэлеевской волн, амплитуды которых соответствуют нормативному значению ускорения грунта 4 м/ ${ }^{2}$, выбраны максимальное растягивающее и максимальное сжимающее напряжения. На основе полученных данных построены зависимости, приведенные на рис. 4.
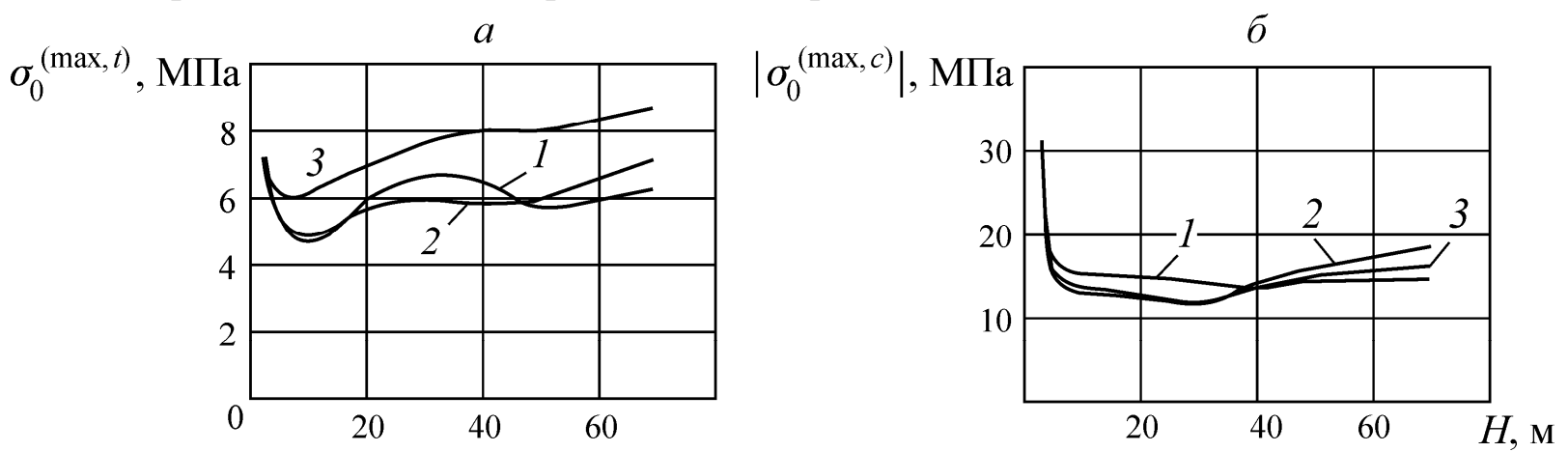

Рис. 4. Зависимости максимальных растягивающих и сжимающих напряжений, которые могут возникнуть в обделке тоннеля при землетрясении, от глубины заложения

Видно, что максимальные растягивающие напряжения возрастают с увеличением глубины заложения тоннеля, при этом, когда тоннель расположен в грунтах с коэффициентом Пуассона $v_{0}=0.4$, напряжения в обделке тоннеля существенно выше, чем в остальных случаях. Зависимость максимальных сжимающих напряжений от глубины заложения имеет минимум на глубине 30-40 м, что соответствует $\sim 1 / 4$ длины поперечной волны. При дальнейшем увеличении глубины заложения напряжения возрастают.

Очевидно, что приведенные заключения справедливы только для однородного массива грунта. При наличии мощных слоев с сильно отличающимися деформационными характеристиками зависимости максимальных напряжений в обделке тоннеля от глубины заложения будут иными.

\section{ВЫВОДЫ}

Использование нового метода расчета обделок тоннелей мелкого заложения на сейсмические воздействия землетрясений позволяет определять опасные сечения подземной конструкции, в которых возникновение трещин при землетрясении наиболее вероятно.

Обделки тоннелей мелкого заложения при землетрясении испытывают, преимущественно, горизонтальное сжатие, что обусловлено особенностями формирования напряженного состояния грунтового массива при распространении в нем сейсмических волн.

Зависимости максимальных напряжений, возникающих в обделке тоннеля при землетрясении, имеют достаточно сложный характер. Тем не менее, можно с уверенностью утверждать, что тоннели мелкого заложения в большей степени подвержены действию поперечных волн и волн Рэлея, чем подземные сооружения, расположенные на значительной глубине. 


\section{СПИСОК ЛИТЕРАТУРЫ / REFERENCES}

1. Owen G. N. and Scholl R. E. Earthquake engineering of large underground structures. Report no. FHWA (RD-80)195. Federal Highway Administration and National Science Foundation. 1978.

2. Dowding C. H. and Rozen A. Damage to rock tunnels from earthquake shaking, J. Geotech. Eng. Div., ASCE 104 (GT2.), 1981, pp. 175-191.

3. Wang J.-N. Seismic Design of Tunnels: a state-of-the-art approach. Monograph. New York, Parsons, Brinckerhoff, Quade and Douglas Inc, 1993.

4. Instructions for accounting seismic effects in the design of mountain transport tunnels VSN 193-81, Moscow, 1982. (in Russian) [Инструкция по учету сейсмических воздействий при проектировании горных транспортных тоннелей ВСН 193-81. - М.: Минтрансстрой, 1982.]

5. Building regulations 268. 1325800.2016. Transport facilities in seismic regions. Design rules, Moscow, 2017, 170 pp. (in Russian). [Строительные правила 268. 1325800.2016. Транспортные сооружения в сейсмических районах. Правила проектирования. — М., 2017. - 170 с.]

6. Fotieva N. N. Design of underground structures support in seismically active regions, Moscow, Nedra, 1980. (in Russian) [Фотиева Н. Н. Расчет крепи подземных сооружений в сейсмически активных районах. — М.: Недра, 1980. — 222 c.]

7. Fotieva N. N. and Shelepov N. V. Seismic design of shallow tunnels' linings, Mining Information Analytical Bulletin, 2000, no. 3, pp. 26-30. (in Russian) [Фотиева Н. Н., Шелепов Н. В. Расчет обделок тоннелей мелкого заложения на сейсмические воздействия // ГИАБ. - 2000. — № 3. - С. 26-30.]

8. Fotieva N. N., Bulychev N. S., Sammal A. S., and Deev P. V. Mathematical modeling of stress state of shallow tunnel lining subjected to action of long seismic waves, Izv. TulGU, Ser. Geomechanics. Mechanics of underground structures, 2005, vol. 3, pp. 199-209. (in Russian) [Фотиева Н. Н., Булычев Н. С., Саммаль А. С., Деев П. В. Математическое моделирование напряженного состояния обделок тоннелей мелкого заложения при распространении в массиве длинных сейсмических волн // Изв. ТулГУ. Сер. Геомеханика. Механика подземных сооружений. - 2005. — Вып. 3. — С. 199-209.]

9. Deev P. V. and Petrukhin M. A. Seismic calculation of shallow tunnels, Izv.TulGU, Ser. Earth Sciences, 2018, vol. 3, pp. 212-221. (in Russian) [Деев П. В., Петрухин М. А. Сейсмический расчет тоннелей мелкого заложения // Изв. ТулГУ. Сер. Науки о Земле. - 2018. - Вып. 3. - С. 212 -221.] 